\title{
Strict Bounds on Franson Inequality
}

\author{
Lev Vaidman and Ori Belkind \\ School of Physics and Astronomy, Raymond and Beverly Sackler Faculty of Exact Sciences, \\ Tel-Aviv University, Tel-Aviv 69978, Israel.
}

()

\begin{abstract}
An inequality, recently proposed by Franson [Phys. Rev. A 54, 3808 (1996)] is analyzed and improved. The inequality connects the change of the expectation value of an observable with the uncertainty of this observable. A strict bound on the ratio between these two quantities is obtained.
\end{abstract}

In a recent paper Franson [1] developed a quantitative expression which characterizes the fact that a quantum observable cannot change its expectation value without having some quantum uncertainty. The simplest explanation of this property is that in order for a quantum system to go from one eigenvalue of an observable to another it must evolve through a superposition of the eigenstates which is characterized by nonzero uncertainty of the observable. Franson proposed to consider the change of an observable $Q$ after it has been measured such that the initial state of the system at time $t=0$ is an eigenstate of the observable. Then, at any moment of time $t$ in the period $\tau_{1}$

$$
\tau_{1}=\frac{\hbar}{\sqrt{2} \Delta E}
$$

where $\Delta E$ is the energy uncertainty of the system, the following inequality is fulfilled:

$$
|\delta\langle Q\rangle| \leq \Delta Q
$$

Here, $\Delta Q=\sqrt{\left\langle\Psi(t)\left|Q^{2}\right| \Psi(t)\right\rangle-\langle\Psi(t)|Q| \Psi(t)\rangle^{2}}$ is the uncertainty at time $t$ and $\delta\langle Q\rangle=\langle\Psi(t)|Q| \Psi(t)\rangle-$ $\langle\Psi(0)|Q| \Psi(0)\rangle$ is the change in the expectation value.

If an inequality expresses a basic physical law, there must be cases where it is saturated; if not, it must be replaced by a stricter inequality which does saturate. The Franson inequality as it is presented in his paper cannot reach the equality (except at $t=0$ ). The purpose of this paper is to improve Franson's bound such that the revised inequality cannot be replaced by any stricter inequality.

There are two elements which we improve in the Franson inequality. First, we replace his approximate calculations by exact calculations and obtain, instead of (1), a larger period of time $\tau_{2}$ for which the inequality (2) is fulfilled. Then, for time $t=\tau_{2}$ the inequality can be saturated. However, for any intermediate time, $t \in\left(0, \tau_{2}\right)$, the inequality cannot be saturated. In order to correct this we introduce time explicitly into the the inequality.

\footnotetext{
${ }^{1}$ Recently, $\mathrm{Yu}$ [2] described an analog of this time-energy inequality for position and momentum.
}

The Franson inequality is closely connected with a certain type of time-energy inequality which constrains the time of the evolution of a system to an orthogonal state:

$$
t \geq \frac{h}{4 \Delta E} .
$$

Although it has a similar form, it is conceptually different from the Heisenberg uncertainty relations for position and momentum. ${ }^{1}$ There are many derivations of this results. As far as we know the first derivation was given by Mandelstam and Tamm [3], and a very simple derivation can be found in Vaidman [4]. We will apply this result for deriving a strict bound on the Franson inequality. A more general form of the time-energy inequality which we will need here is:

$$
|\langle\Psi(t) \mid \Psi(0)\rangle| \geq \cos \left(\frac{\Delta E t}{\hbar}\right),
$$

which is valid for $t \in\left[0, \frac{\pi \hbar}{2 \Delta E}\right]$ where $\Delta E$ is the energy uncertainty of the system.

Let us turn to finding the optimal bound on the period of time for which the Franson inequality (2) is fulfilled. This problem is equivalent to finding the minimal (nonzero) time for which $|\delta\langle Q\rangle|=\Delta Q$. Without loss of generality we can assume that the eigenvalue of $Q$ at the initial time is zero, i.e. $|\Psi(0)\rangle=|Q=0\rangle \equiv|0\rangle$. At time $t$ the state of the system can be expressed as

$$
|\Psi(t)\rangle=\alpha|0\rangle+\beta|1\rangle
$$

where $|1\rangle$ is orthogonal to $|0\rangle$ and $\beta$ is real and positive. Then,

$$
\begin{aligned}
\delta\langle Q\rangle & =\beta^{2}\langle 1|Q| 1\rangle, \\
\Delta Q & =\sqrt{\beta^{2}\left\langle 1\left|Q^{2}\right| 1\right\rangle-\beta^{4}\langle 1|Q| 1\rangle^{2}} .
\end{aligned}
$$

From $|\delta\langle Q\rangle|=\Delta Q$ follows:

$$
\beta^{2}=\frac{1}{2} \frac{\left\langle 1\left|Q^{2}\right| 1\right\rangle}{\langle 1|Q| 1\rangle^{2}}
$$

Since $\langle 1|Q| 1\rangle^{2}$ is always smaller or equal to $\left\langle 1\left|Q^{2}\right| 1\right\rangle$, the minimal value for $\beta$ is $\sqrt{\frac{1}{2}}$. For this value of $\beta$ we obtain: 


$$
|\langle\Psi(t) \mid \Psi(0)\rangle|=|\alpha|=\sqrt{1-\beta^{2}}=\cos \frac{\pi}{4} .
$$

From the time-energy relation (4) it follows that the minimal time (which corrects Franson's bound (1)) is:

$$
\tau_{2}=\frac{\pi \hbar}{4 \Delta E} .
$$

Since $1 / \sqrt{2}<\pi / 4$, the time limit we found is indeed larger than that of Franson (Eq. 11). Note, that this is half the minimal time of an evolution to an orthogonal state.

Consider an example of a spin- $\frac{1}{2}$ particle precessing in magnetic field. Let us assume that the initial state is $|\uparrow\rangle$, the "up" state in the $z$ direction, and that the magnetic field is in the $y$ direction. Then, the time evolution is:

$$
|\Psi(t)\rangle=\cos \left(\frac{\Delta E t}{\hbar}\right)|\uparrow\rangle+\sin \left(\frac{\Delta E t}{\hbar}\right)|\downarrow\rangle .
$$

Therefore,

$$
\begin{aligned}
\delta\left\langle\sigma_{z}\right\rangle & =\cos \left(\frac{2 \Delta E t}{\hbar}\right)-1, \\
\Delta \sigma_{z} & =\sin \left(\frac{2 \Delta E t}{\hbar}\right) .
\end{aligned}
$$

Thus we see that $\left|\delta\left\langle\sigma_{z}\right\rangle\right| \leq \Delta \sigma_{z}$ for $t \leq \tau_{2}$. The equality is reached for $t=\tau_{2}$.

The same bound is obtained for any other two-level system or even for any system with higher-dimensional Hilbert space which spans only a two-dimensional Hilbert space during the evolution. Introducing any additional state will invariably increase the minimal time for reaching the equality $|\delta\langle Q\rangle|=\Delta Q$.

It is interesting to consider the connection between $\delta\langle Q\rangle$ and $\Delta Q$ when we do not impose the initial condition $\Delta Q=0$. We ask what is the minimal period of time during which the change in the expectation value reaches the value of the maximal uncertainty during this period of time.

Consider our example of a spin- $\frac{1}{2}$ particle. A simple analysis shows that the minimal time to reach the equality $|\delta\langle Q\rangle|=\Delta Q$ is obtained for the evolution between the states

$$
\begin{aligned}
\left|\Psi_{i n}\right\rangle & =\cos \left(\frac{\pi}{6}\right)|\uparrow\rangle+\sin \left(\frac{\pi}{6}\right)|\downarrow\rangle, \\
\left|\Psi_{f}\right\rangle & =\cos \left(\frac{\pi}{3}\right)|\uparrow\rangle+\sin \left(\frac{\pi}{3}\right)|\downarrow\rangle .
\end{aligned}
$$

This time is

$$
\tau_{3}=\frac{\pi \hbar}{6 \Delta E} .
$$

In this case $\left|\delta\left\langle\sigma_{z}\right\rangle\right|=\max \left(\Delta \sigma_{z}\right)=1$ and the maximum uncertainty is obtained at the middle point of the evolution.
However, an example in which only two states are involved is not the optimal one. A system of $N$ spin- $\frac{1}{2}$ particles precessing in a magnetic field yields a smaller time for reaching the equality $\left|\delta\left\langle\sum_{i}\left(\sigma_{z}\right)_{i}\right\rangle\right|=\Delta\left(\sum_{i}\left(\sigma_{z}\right)_{i}\right)$. The minimal time for this is:

$$
\tau_{4}=\sqrt{N} \arcsin \left(\frac{1}{2 \sqrt{N}}\right) \frac{\hbar}{\Delta E} .
$$

At the limit of $N \rightarrow \infty$ we reach the minimal time

$$
\tau_{5}=\frac{\hbar}{2 \Delta E} \text {. }
$$

The same minimal time is obtained in the most natural example of comparison between $\delta\langle Q\rangle$ and $\Delta Q$. Consider a free particle of mass $m$ in one dimension in a (minimal uncertainty) Gaussian wave packet which moves with velocity $v$. The velocity is high enough such that we can neglect the spread of the wave-packet. Since $E=p^{2} / 2 m$, for large velocity we obtain

$$
\Delta E \sim \frac{\langle p\rangle \Delta p}{m} .
$$

Then, taking into account the Heisenberg relation for minimal uncertainty, $\Delta x \Delta p=\hbar / 2$, we find the time for which $\delta\langle x\rangle=\Delta x$ :

$$
\frac{\Delta x}{v}=\frac{\Delta x m}{\langle p\rangle}=\frac{\Delta x \Delta p}{\Delta E}=\frac{\hbar}{2 \Delta E} .
$$

The period $\tau_{5}$ is a strict bound for the minimal period of time during which the change in the expectation value reaches the value of the maximal uncertainty. This result can be obtained immediately from the Heisenberg relation:

$$
\Delta E \Delta Q \geq \frac{1}{2}|\langle[H, Q]\rangle|=\frac{\hbar}{2}\left|\frac{d\langle Q\rangle}{d t}\right| .
$$

If $\Delta Q$ is essentially constant during the process, we find that the equality $|\delta\langle Q\rangle|=\Delta Q$ is reached during the time $\tau_{5}$.

The strict bound $\tau_{5}$ and the bounds $\tau_{3}$ and $\tau_{4}$ are bounds for a different problem from the one Franson proposed. The novelty of his inequality is in considering an evolution starting from an eigenstate of an observable. For such a problem only the Franson bound $\tau_{1}$ and the improved bound $\tau_{2}$ are relevant. The bound $\tau_{2}$ is absolute in the sense that we cannot replace it by a smaller value and the inequality (2) would still be fulfilled for the whole period $t \in[0, \tau]$. However, even with this exact bound the inequality cannot be considered as a fundamental one because it cannot be saturated for any time except $t=0$ and $t=\tau_{2}$. In order to find a basic inequality let us return to Eqs. (6) and (7), but now we will not limit ourselves to the equality $|\delta\langle Q\rangle|=\Delta Q$. By dividing the equations we obtain:

$$
\frac{|\delta\langle Q\rangle|}{\Delta Q}=\frac{1}{\sqrt{\frac{\left\langle 1\left|Q^{2}\right| 1\right\rangle}{\beta^{2}\langle 1|Q| 1\rangle^{2}}-1}} .
$$


From the time-energy relation (4) we obtain

$\beta=\sqrt{1-|\langle\Psi(0) \mid \Psi(t)\rangle|^{2}} \leq \sqrt{1-\cos ^{2}\left(\frac{\Delta E t}{\hbar}\right)}=\sin \left(\frac{\Delta E t}{\hbar}\right)$.

Now, taking again into account that $\langle 1|Q| 1\rangle^{2} \leq\left\langle 1\left|Q^{2}\right| 1\right\rangle$ we obtain the new inequality:

$$
\frac{|\delta\langle Q\rangle|}{\Delta Q} \leq \tan \left(\frac{\Delta E}{\hbar} t\right)
$$

This inequality is valid for $t \in\left(0, \frac{\pi \hbar}{2 \Delta E}\right]$. The inequality (24) is a basic law since it cannot be replaced by a stricter inequality. Indeed, the example of a spin- $\frac{1}{2}$ particle precessing in a magnetic field saturates the inequality: it becomes an equality for the whole period $\left(0, \frac{\pi \hbar}{2 \Delta E}\right]$.
It is a pleasure to thank Lior Goldenberg and Aharon Casher for helpful discussions. The research was supported in part by grant $614 / 95$ of the Israel Science Foundation. Part of this work was completed during the 1997 Elsag-Bailey - I.S.I. Foundation research meeting on quantum computation.

[1] J.D. Franson, Phys. Rev. A 54, 3808 (1996).

[2] T. Yu, Phys. Lett. A 223, 9 (1996).

[3] L. Mandelstam and I. Tamm, J. Phys. (USSR) 9, 249 (1945).

[4] L. Vaidman Am. J. Phys. 60, 182 (1992). 